\title{
El tránsito hacia la profesión docente: una experiencia compartida
}

\author{
Ilse Carolina Castro Zavaleta y Tania Itzel Nieto Juárez
}

\section{Resumen}

A lo largo de la historia, las universidades han forjado y desarrollado diversos modelos de formación de sus cuadros docentes. En el caso de la Universidad Nacional Autónoma de México (UNAm), la ayudantía ha sido uno de los modelos que aún continúa vigente y se ha constituido a su vez como la etapa inicial de la carrera académica de varios profesores universitarios. Recuperar este proceso de formación desde la voz de los sujetos involucrados brinda elementos únicos del trabajo docente en la unAm. Así, el presente artículo da cuenta de la experiencia de formación de dos profesoras noveles ${ }^{1}$ del Colegio de Pedagogía, en la Facultad de Filosofía y Letras (FFyL) de la UNAM, quienes se desempeñaron como ayudantes de profesor. Ésta es abordada a través de dos momentos: el desarrollo del rol como ayudantes de profesor y el transcurso de su primer año como profesoras de asignatura.

Palabras clave: profesores noveles, ayudantes, formación universitaria, experiencia pedagógica.

\section{THE TRANSITION FROM ASSISTANT PROFESSOR TO NOVEL PROFESSOR:}

\section{A SHARED EXPERIENCE}

1"Entendemos por profesor novel cualquier profesor universitario que se encuentre en sus tres primeros años de vida profesional" (Benedito, et al., 1995,

p. 176).

\begin{abstract}
Throughout history, universities have forged and developed different training models for their teaching staff. In the case of the National Autonomous University of Mexico (UNAM), the teaching assistant is one of the models that has continued to constitute the initial stage of the academic career of several university professors. Recovering this training process from the point of view of people directly involved in it provides unique elements of the teaching work at unAm. Thus, this article takes into account the training experience of two teachers of the Department of Pedagogy, of the Faculty of Philosophy and Letters (FFyL) of UNAM who worked as teaching assistants. Such episodes are approached in two stages: their development as teaching assistants and their first year as teachers.
\end{abstract}

Keywords: teachers, assistants, university training, pedagogical experience.

Dol: http://doi.org/10.22201/codeic.16076079e.2019.v20n6.a5 


\section{Ilse Carolina Castro Zavaleta}

Licenciada en Pedagogía por la Universidad Nacional Autónoma de México (UnAM). Es profesora de la Licenciatura en Pedagogía de la Facultad de Filosofía y Letras de la unAm y ayudante de investigador del Sistema Nacional de Investigadores. Sus temas de interés son la investigación educativa, la educación superior y la política internacional en educación.

\section{Tania Itzel Nieto Juárez}

tania.nieto@unam.mx

Licenciada en Pedagogía por la UNAM y especialista en entornos virtuales de aprendizaje por la Organización de Estados Iberoamericanos para la Educación, la Ciencia y la Cultura-Virtual Educa-Centro de Altos Estudios Universitarios. Actualmente es profesora de asignatura en el Colegio de Pedagogía de la Facultad de Filosofía y Letras, y estudia el cuarto semestre de la maestría en Pedagogía en la UNAM, con orientación en docencia.

Realizó un semestre de movilidad estudiantil en la Universidad de Barcelona (UB) en la Facultad de Pedagogía y es alumni de la red de estudiantes del Observatori de l'Estudiant de la uB. Sus temas de interés son educación superior, prácticas y formación docente, historia de la educación y la pedagogía de México, e historia de la unam. 


\section{Docencia y formación del profesorado universitario}

La función docente es una de las tareas encomendadas a las instituciones de educación superior junto con la investigación y la difusión de la cultura. Se trata de una práctica social que tiene lugar en un contexto determinado, configurándose de acuerdo a las condiciones sociales, políticas, económicas y disciplinares del espacio en el que se ejerce.

En el cumplimiento de esta labor los profesores son actores cruciales, puesto que son los agentes sustanciales en el proceso formativo de los futuros profesionales. Así el docente puede ser "considerado como un sujeto transformador que trasciende la individualidad y se ubica como un agente social" (Hernández, 2016: 260), siendo a su vez transformado institucional y socialmente.

A través de la historia de la profesión el papel del profesor universitario se ha venido modificando en cuanto al deber ser. De esta manera, desde hace ya algunas décadas se ha considerado que:

El profesorado universitario [...] debe ser reflexivo, crítico, competente en el ámbito de su disciplina, capacitado para ejercer la docencia y para realizar actividades de investigación. Debe intentar desarrollar una actividad docente comprometida con la idea de potenciar el aprendizaje de los estudiantes y contribuir a la mejora de la sociedad (Benedito, et al., 1995: 119).

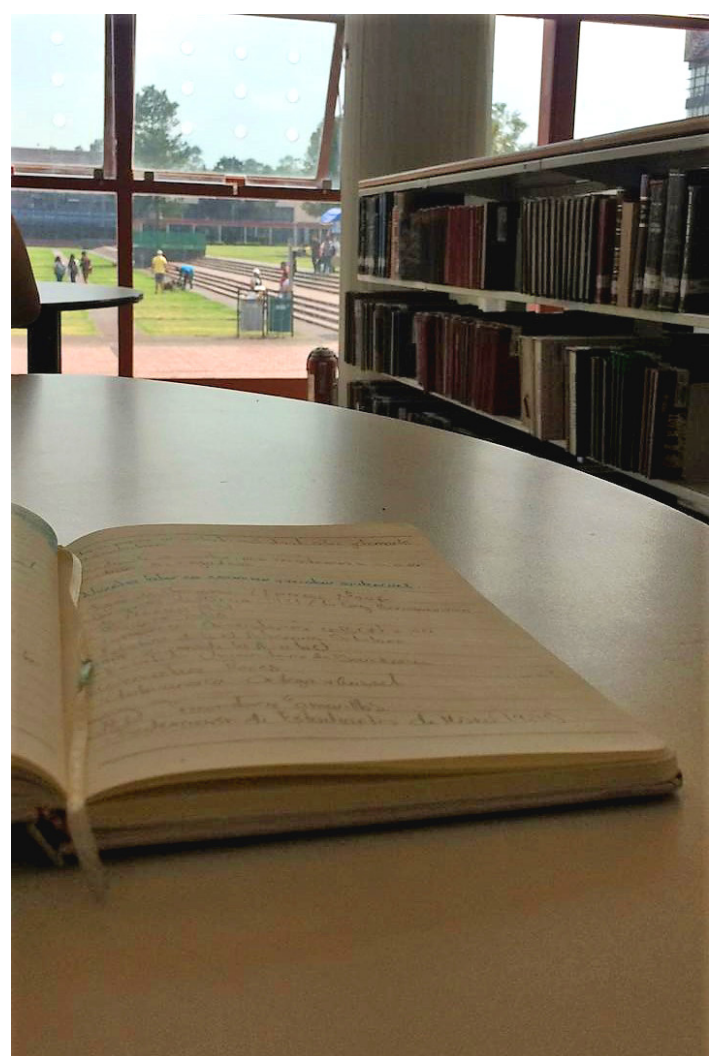

En este sentido, la labor que realizan día a día se constituye como una práctica específica y compleja, ya que la docencia no implica únicamente el trabajo en el aula, supone además una serie de actividades previas y posteriores a esa acción, relacionadas con la actividad académica (Zabalza, 2007). Además del hecho de que para ejercer la docencia universitaria no es suficiente con ser experto en la disciplina, hay que poseer un saber pedagógico. Por supuesto la primera es una condición básica, no se puede enseñar lo que no se conoce, pero no por conocer la disciplina a profundidad se sabe enseñarla; por ello la formación docente es condición necesaria para el ejercicio profesional de la enseñanza.

En este marco nos surgen las siguientes preguntas: ¿Cómo debería formarse a los docentes? ¿Qué características posee un profesor que ha sido formado para el ejercicio docente? ¿Cómo impacta la formación en la práctica docente? ¿Cuál es la pertinencia de la formación para los docentes, los estudiantes, la institución y la propia sociedad? Resolver estas cuestiones rebasa el objetivo de este artículo, sin embargo, nos centraremos en aclarar desde dónde estamos entendiendo el concepto de formación. 
Tabla 1. Ámbitos del profesor que influyen en su actuar profesional.
Retomamos, entonces, el concepto derivado de la tradición alemana: bildung (formación), el cual hace referencia al "trabajo sobre sí mismo, cultivo de los talentos para el perfeccionamiento propio" (Fabre, 2011: 216). A su vez, bildung también implica libertad; por ello, al formar a un sujeto se pretende constituirlo como un ser autónomo:

El individuo formado es libre porque se le ha conferido un grado de autonomía en el que puede trazar su propio rumbo; decide avanzar o retroceder [...]. El sujeto encuentra la libertad y su autonomía al poseer un bagaje de escenarios de actuación amplio y de los que puede echar mano para dar respuesta a sus necesidades (Hernández, 2016: 269).

Un modelo basado en este concepto debe considerar al menos tres ámbitos del profesor que, en sincronía, influyen en su actuar profesional: el histórico, el social y el institucional (ver tabla 1).

\begin{tabular}{|c|c|c|}
\hline Sujeto histórico & Sujeto social & Sujeto institucional \\
\hline $\begin{array}{l}\text { El docente al haber sido } \\
\text { estudiante, y en su caso } \\
\text { ayudante de profesor, } \\
\text { tiene ya un referente } \\
\text { previo de lo que es la } \\
\text { docencia, así, "desde las } \\
\text { primeras ocasiones que } \\
\text { se enfrenta a un grupo, } \\
\text { su actuar se encuentra } \\
\text { definido por la imitación } \\
\text { de la actuación de } \\
\text { sus propios maestros, } \\
\text { los cuales se vuelven } \\
\text { referente para su propio } \\
\text { estilo de docencia" } \\
\text { (Hernández, 2016: 258). }\end{array}$ & $\begin{array}{l}\text { Implica considerar que } \\
\text { el docente no ejecuta su } \\
\text { práctica únicamente desde } \\
\text { lo individual, sino que } \\
\text { está enmarcada en una } \\
\text { colectividad que se desarrolla } \\
\text { en un contexto temporal } \\
\text { y geográfico específico. } \\
\text { De hecho, "se considera a } \\
\text { la formación del maestro } \\
\text { como una tarea social que } \\
\text { se ve influida por los actores } \\
\text { cercanos a su quehacer" } \\
\text { (Hernández, 2016: 260). Es } \\
\text { decir, se configura un diálogo } \\
\text { entre el profesor y demás } \\
\text { comunidad educativa y } \\
\text { social. }\end{array}$ & $\begin{array}{l}\text { Cada institución tiene una } \\
\text { concepción de la figura } \\
\text { y funciones docentes. } \\
\text { Aunado a ello cada entidac } \\
\text { ha desarrollado a lo largo } \\
\text { de su devenir un modelo } \\
\text { de formación docente } \\
\text { particular. } \\
\text { "La formación con una } \\
\text { visión institucional se } \\
\text { establece como una } \\
\text { manera de alineación } \\
\text { entre el sujeto (formado) } \\
\text { y la institución (formador)" } \\
\text { (Hernández, 2016: 261). }\end{array}$ \\
\hline
\end{tabular}

Así, se puede afirmar que la formación docente no es meramente una "capacitación" o "entrenamiento" para desempeñar la tarea, sino que implica a su vez la autoformación del sujeto en una comunidad educativa con códigos, formas y saberes específicos.

\section{Figura del ayudante de profesor: formación práctica}

Desde el surgimiento de las universidades en la Edad Media, la formación de los docentes ha estado a cargo de los catedráticos más reconocidos, éstos contaban con ayudantes en sus clases, quienes posteriormente se fueron integrando al gremio hasta lograr obtener una plaza y continuar el ciclo de formación.

En nuestro país, las narraciones que se han hecho respecto a las cátedras de la Real y Pontificia Universidad de México durante la época colonial hacen 
alusión a la figura y tareas del ayudante de algunos catedráticos novohispanos, un modelo de formación que se conservó a través del devenir de la institución.

Hacia 1910, con la fundación de la Universidad Nacional de México la formación del docente universitario se planteó bajo un modelo profesionalizante, siendo la Escuela Nacional de Altos Estudios el espacio dedicado a ello, sin embargo, en años posteriores, dicho modelo fue suprimido.

En 1945, con la publicación de la Ley Orgánica de la UNAM, aún vigente, se establece entre las finalidades de la Universidad la formación de profesores universitarios:

Art. 1: La Universidad Nacional Autónoma de México es una corporación pública - organismo descentralizado del Estado- dotada de plena capacidad jurídica y que tiene por fines impartir educación superior para formar profesionistas, investigadores, profesores universitarios y técnicos útiles a la sociedad; organizar y realizar investigaciones, principalmente acerca de las condiciones y problemas nacionales, y extender con la mayor amplitud posible los beneficios de la cultura (UNAM, 1945).

Posterior a ello, respecto al trabajo de los académicos la Universidad han elaborado distintos documentos que norman tal actividad. La normatividad vigente es el Estatuto del Personal Académico (EPA), elaborado en la década de los setenta. En éste se reconoce la figura de ayudantes de profesor y de investigador, definiéndolos como aquellos que "auxilian a los profesores y los investigadores en sus labores" (UNAM, 1988). También menciona que dicho nombramiento es temporal y establece que "la ayudantía debe capacitar al personal para el desempeño de funciones docentes, o de investigación" (UNAM, 1988).

Así, la naturaleza de la ayudantía es dual, es decir, de apoyo y también formativa; ya que, al apoyar al profesor, el ayudante se implica de manera directa en las labores docentes, configurándose como un proceso formativo in situ. Es así que la ayudantía puede considerarse como una etapa de inducción, como un proceso de formación inicial, la cual:

[...] debe al menos suministrar las bases para adquirir una determinada "mirada pedagógica", proporcionar unos instrumentos y establecer un contacto con una actividad que los y las aspirantes sólo conocen por haberla vivido como receptores, pero sin haber reflexionado sobre ella y desde ella (Imbernón, 2019: 4).

\section{El inicio de la experiencia compartida: la ayudantía}

El Estatuto del Personal Académico de la unam señala que "la adscripción de los ayudantes se hará de acuerdo con las bases que fije al efecto el consejo técnico o interno en su caso, de la dependencia respectiva" (UNAM, 1988). En cumplimiento de lo anterior, para el período 2011-2018, la Facultad de Filosofía y Letras estableció los Lineamientos para el nombramiento y la regulación de las actividades de ayudantes de profesor (FFy L, 2011). En dicho documento se presentan las características del nombramiento de ayudante de profesor, los requisitos y 
${ }^{2}$ El 26 de abril de 2019, el Consejo Técnico de la fFyL aprobó los nuevos Lineamientos de solicitud, aprobación y seguimiento de ayudantes de profesor. el proceso para la contratación, así como las obligaciones que adquieren tanto el docente como el ayudante. ${ }^{2}$ Con respecto al nombramiento se establecen dos niveles indicados con las letras " $\mathrm{A}$ " $\mathrm{y}$ " $\mathrm{B}$ ", estos son asignados principalmente con base en el avance de créditos. Sus modalidades pueden ser por efectos curriculares o de remuneración económica, y la duración es de un semestre con posibilidad de ser renovado hasta seis veces.

A continuación, narramos en tres momentos nuestra experiencia como ayudantes de profesor que abarcó desde el segundo semestre de 2014 hasta el primer semestre de 2018.

\section{Elección}

Son múltiples los factores para la elección del ayudante de profesor, uno de ellos es el desempeño y compromiso académico que el estudiante demuestra a lo largo de su trayectoria de formación (constancia, participación, exposición de temas, conducción de actividades grupales y, en ocasiones, propuesta de recursos y materiales). Cabe mencionar que la experiencia que aquí presentamos tiene como punto inicial el haber sido alumnas de los profesores con los que después colaboramos, lo cual permitió que ellos identificaran en nosotras lo antes mencionado, al tiempo que ambas fuimos identificando la manera en la que ellos trabajan en el aula.

El proceso formal de invitarnos a colaborar como ayudantes provino del docente titular, ya que son ellos quienes realizan la solicitud de ayudantía ante el Consejo Técnico de la Facultad. Sin embargo, desde que fuimos sus estudiantes manifestamos interés por una posible colaboración con ellos.

\section{Desarrollo}

Una vez que el registro procede ante el Consejo Técnico, comienza el trabajo del curso. Así, en un primer momento los docentes nos compartieron su propuesta de trabajo y el programa de la asignatura, para tener conocimiento de los mismos, y nos solicitaron apoyo para su enriquecimiento considerando la selección de estrategias, técnicas, recursos y materiales diversos, la búsqueda de bibliografía de la asignatura, y la definición de la evaluación y los criterios correspondientes.

Esta actividad permite visualizar diversas opciones tanto de textos especializados como de recursos y materiales didácticos, los cuales son propuestos por ambas partes y, finalmente, los más adecuados para la clase son seleccionados por parte del docente titular quien ya cuenta con experiencia en la materia. Desde nuestra posición, se trata de una tarea que se va afinando con el transcurso de los semestres y que a su vez propicia un reconocimiento de autores y obras fundamentales sobre los temas de la asignatura, generando así un mayor conocimiento que nos permitió ir construyendo un discurso sistemático respecto a ésta. 
Cabe mencionar que a lo largo de nuestra formación universitaria interactuamos con los ayudantes de nuestros profesores en diversas clases, con ello, tuvimos conocimiento de las implicaciones del rol que desempeñaban; así, esta acción se constituyó como un referente al inicio de nuestra actividad como tales. Sin embargo, no pudimos dejar de preguntarnos cuáles eran las funciones específicas de un ayudante de profesor y hasta dónde podíamos intervenir en la planeación de las sesiones y en cada una de las clases.

En nuestra primera sesión frente al grupo, los docentes nos presentaron como sus ayudantes de profesor, acción que nos posicionó frente al grupo y dio la pauta para comenzar a desempeñar nuestro nuevo rol.

La relación con los estudiantes generalmente es más cercana pues la brecha generacional es mínima, sin embargo, consideramos que ésta debe irse construyendo en un marco de respeto y acompañamiento. Asimismo, el mostrar un compromiso con la clase a través de la asistencia, puntualidad, participación activa, organización, asesoría, entre otras, afianzó nuestro rol y llegó a contribuir a la formación de los estudiantes.

En cada una de las sesiones estuvimos presentes para apoyar al docente en diversas actividades que tienen que ver con el desarrollo de la clase, a su vez, fuimos realizando participaciones que buscaban aportar al abordaje de los distintos temas, al tiempo que observábamos y aprendíamos cómo se desenvolvía el docente en el aula, su lenguaje verbal y no verbal, la comunicación que establecía con los alumnos, la forma en la que presentaba los temas, los elementos que utilizaba para generar distintas dinámicas en el grupo, su aproximación a la trayectoria de los estudiantes, y las formas de evaluación.

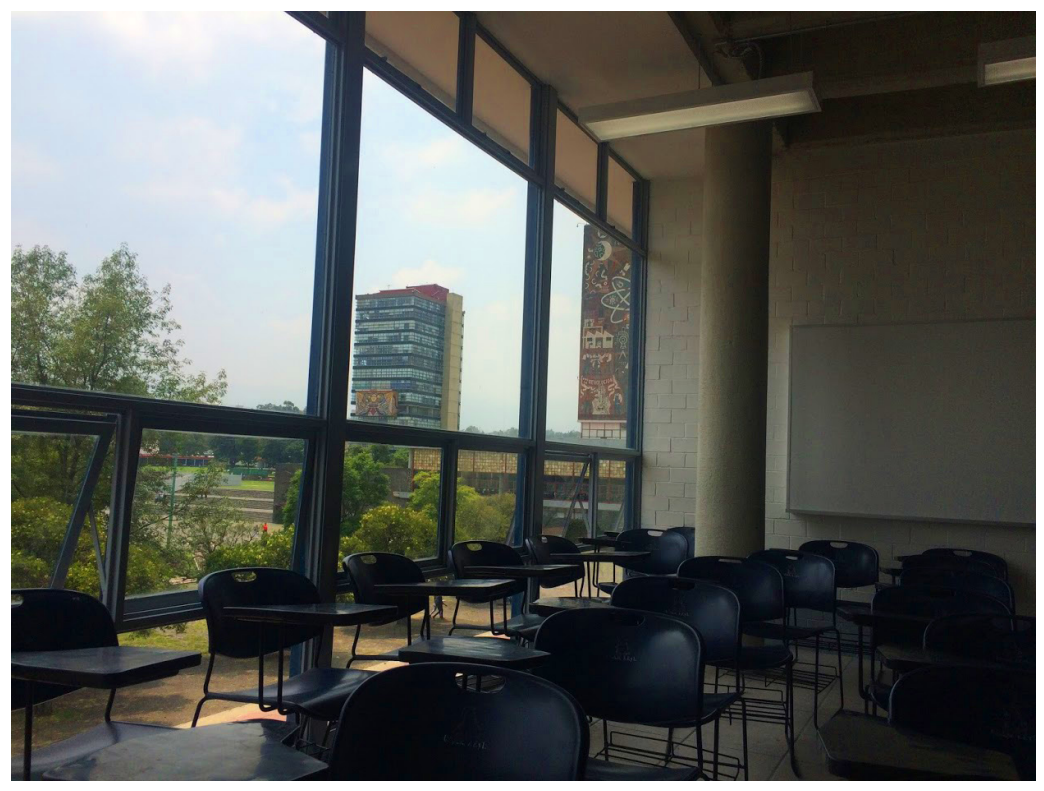


Dado que los lineamientos de la fFy indican que los ayudantes de profesor deben impartir entre 15\% y 25\% de las clases, se nos brindó la oportunidad de dar, al menos, dos temas del programa de la asignatura. Esta labor implicó la planeación de la sesión, la búsqueda de información, así como la construcción de recursos y actividades que utilizaríamos. Es necesario resaltar que partimos de la idea de que conducir una clase es más que una exposición del tema, pues implica constituir un ambiente formativo, dialogar con los alumnos, reflexionar y construir aprendizajes. Todo ello, estuvo acompañado de las observaciones elaboradas por los docentes titulares. De esta manera, conforme desarrollamos la experiencia a lo largo de los ciclos, fuimos configurando nuestra identidad como docentes.

Otro de los elementos formativos es el conocimiento de las actividades académico-administrativas de la entidad, lo cual nos brindó elementos para orientar a los estudiantes respecto a diversos temas relacionados con su trayectoria escolar y gestiones a realizar en la Facultad. Asimismo, fuimos conociendo a otros actores que desempeñaban la misma función que nosotras en asignaturas distintas, a los docentes cercanos al profesor titular, e incluso a otros de una disciplina diferente a la propia, lo que propició que fuéramos constituyendo redes.

Cabe destacar que la formación docente se expresa formalmente por un período de tiempo; por ello, el que está participando como ayudante siempre tiene la expectativa de un lugar propio, de tener a su cargo una asignatura, una vez concluida la formación. Aunque en ocasiones esto no tenga lugar en la misma institución en la que se recibió la formación.

\section{Cierre}

A lo largo de nuestro desempeño como ayudantes, los profesores fueron nuestros modelos de referencia y a su vez ellos nos brindaron la confianza de participar de manera activa en la planeación y desarrollo de cada una de las sesiones, así como en la evaluación de los estudiantes, lo cual nos permitió configurar una idea global de los momentos, tareas y recursos que implica desempeñarse como un docente universitario.

En el último año como ayudantes de profesor nos sentimos con mayor conocimiento y habilidades para desempeñarnos frente al grupo, hecho que fue confirmado por los profesores titulares. Finalmente, justo al concluir la última renovación del nombramiento que ostentábamos, fuimos invitadas a participar como profesoras de asignatura. 
realizamos una valoración de cada una de ellas y vamos generando ajustes a nuestro trabajo. Asimismo, la realimentación por parte de los estudiantes es fundamental, pues son ellos quienes observan nuestro desempeño, y es importante tomar en cuenta sus apreciaciones. A su vez, confirmamos la importancia de conocer a nuestros estudiantes y de mostrarles los amplios, pero focalizados, alcances de la Pedagogía y la responsabilidad social que implica constituirse como un profesional de la educación.

Por otrolado, hemosobservado que nuestra edad no llega aser una fortaleza, pues muchos estudiantes al vernos tan cercanas generacionalmente dudan de los saberes y habilidades que poseemos, tienen múltiples incertidumbres, y consideran que al tener poca experiencia "no seremos buenas docentes". Además, a diferencia de los profesores que cuentan con amplia trayectoria, en nuestro caso hay pocas referencias sobre nuestro trabajo, puesto que recién comenzamos con la construcción de nuestra carrera académica. No obstante, al finalizar el curso fueron los propios estudiantes quienes reconocieron nuestra labor como docentes.

En este sentido, el ganarse la aceptación en la comunidad académica es de suma relevancia, ya que al ser un nuevo miembro en el gremio hay que irse construyendo un lugar. Asimismo, parte de ello es continuar con la formación universitaria disciplinar y pedagógica, pero también participar en actividades de investigación y difusión del conocimiento, así como estar cerca de la generación de conocimientos para poder después compartirlos a quienes comienzan a formarse como profesionales.

Aunado a lo anterior, durante este tiempo nos hemos percatado de que el status de docente también tiene un impacto importante entre otros miembros de la comunidad universitaria, ya que al decir "soy profesora" el trato hacia nuestra persona es con mayor respeto.

\section{Reflexión final}

Compartir experiencias es considerado un proceso de aprendizaje y de construcción de conocimientos de manera conjunta a partir de la socialización de los logros y retos a los que el otro se ha enfrentado. Así, escribir sobre el proceso formativo que hemos seguido nos permitió realizar una reflexión profunda sobre nuestro inicio en el desarrollo de una carrera académica, considerando el proceso formativo y el trabajo de estos cuatro años de experiencias docentes.

Desempeñarse como ayudante de profesor se configuró como nuestra primera aproximación formal al ejercicio docente. En esta experiencia los maestros con los que colaboramos fueron pieza fundamental en la posición que ahora ocupamos, ya que nos brindaron su confianza y la oportunidad para colaborar con ellos en su clase, tuvieron siempre una apertura a incorporar nuestras propuestas y compartieron con nosotras to dos sus saberes, guiándonos 
en la construcción de nuestra propia definición de docencia y de lo que implica ser profesoras universitarias.

De hecho, consideramos que haber sido formadas a través de este modelo enriqueció en gran medida nuestro conocimiento y recursos tanto disciplinares como didácticos, habilitándonos para desempeñar el nombramiento que ahora tenemos. En cada sesión llevamos al aula nuestras experiencias profesionales, de formación en el posgrado y del desempeño como ayudantes de profesor. Finalmente, queda mencionar que el desarrollo de los cursos en este primer año como profesoras de asignatura ha sido toda una experiencia colmada de aprendizajes, autocrítica y reflexión, en la que concebimos nuestro trabajo como un espacio para compartir y construir saberes en favor de los estudiantes y la Pedagogía.

Aunado a ello, tenemos claro que debemos continuar con nuestra formación disciplinar y en materia docente, con la reflexión sistemática de nuestra práctica y esforzándonos de manera constante para ir mejorándola; teniendo presente que la carrera académica implica constancia, disciplina y compromiso con la institución, los estudiantes y nosotras mismas.

\section{Referencias}

* Benedito, V. et al. (1995). La formación universitaria a debate. Barcelona: Universitat de Barcelona.

* Domingo, A. (2014). ¿Qué es la práctica reflexiva? Recuperado de: https:// practicareflexiva.pro/que-es-la-practica-reflexival.

* Fabre, M. (2011). Experiencia y formación: la Bildung. Revista Educación y Pedagogía, 23(59), 215-225).

* fFyl (2011). Lineamientos para el nombramiento y la regulación de las actividades de ayudantes de profesor [documento aprobado por el Consejo Técnico de la Facultad de Filosofía y Letras el 15 de noviembre de 2011]. Recuperado de: http:// datosabiertos.unam.mx/FFyL:RU-UNAM:56227.

* Hernández, S. (2016). El docente como sujeto de formación. En F. Monroy (coord.), Temas de formación docente. Reflexiones, diálogos y propuestas (pp. 258-271). México: CAPUB.

* Imbernón, F. (2019). Presentación. Las buenas prácticas en la formación inicial del profesorado. En G. Cordero, D. Cevallos y K. Rivera (Coords.), Buenas prácticas en formación inicial del profesorado (pp. 4-6). Valencia: Institut de Creativitat i Innovacions Educatives de la Universitat de València. Recuperado de: https://www. toinn.org/wp-content/uploads/2019/04/Toinn-buenas-practicas-formacioninicial-profesorado.pdf.

*ünnermann, C. (2012). Decálogo del docente universitario. Universidades, 54, (4445). Recuperado de: http://www.redalyc.org/articulo.oa?id=37331243005. 
* unam (2013). Estatuto del Personal Académico de la Universidad Nacional Autónoma de México. México: unam. Recuperado de: http://www.aapaunam.mx/assets/ estatuto-del-personal-academico-de-la-unam2.pdf.

- unam (1945). Ley Orgánica de la Universidad Nacional Autónoma de México. México: unAm. Recuperado de: http://abogadogeneral.unam.mx/legislacion/abogen/ documento.html?doc id=15.

* Zabalza, M. (2007). La enseñanza universitaria. El escenario y sus protagonistas. Madrid: Narcea.

\section{Cómo citar este artículo}

* Castro Zavaleta, Ilse Carolina y Nieto Juárez, Tania Itzel (2019). El tránsito hacia la profesión docente: una experiencia compartida. Revista Digital Universitaria (RDU). Vol. 20, núm. 6 noviembre-diciembre. Dol: http://doi.org/10.22201/ codeic.16076079e.2019.v20n6.a5.

Recepción: 25/07/2019. Aprobación: 19/09/2019 